\title{
Transient Analysis of Two-Dimensional State Markovian Queuing Model with Multiple Working Vacations and Non-Exhaustive Service
}

\author{
Indra \\ Associate Professor \\ Department of Statistics \& Operational \\ Research, \\ Kurukshetra University Kurukshetra, \\ Haryana, INDIA
}

\author{
Ruchi \\ Research Scholar \\ Department of Statistics \& Operational \\ Research, \\ Kurukshetra University Kurukshetra, \\ Haryana, INDIA
}

\begin{abstract}
In the present paper, Two-dimensional state time dependent probabilities along with some interesting particular cases are obtained for single server Markovian queuing system where the service mechanism is Non-exhaustive i.e. the server may go on vacation even if there are some customers waiting for service and during a vacation (working) period the server is allowed to do an alternative job at a different rate. The interarrival time, service time, working vacation time and availability time of the server are assumed to be exponentially distributed. Sample computational representations of the solution are developed and results of a simple computation are provided and presented graphically. Finally some particular cases are derived there from.
\end{abstract}

\section{Keywords}

Markovian Queueing system - Multiple Working vacation Non-Exhaustive Service - Laplace transform

\section{INTRODUCTION}

Vacation models had been the subject of interest to queue theorists of deep study in recent years because of their applicability and theoretical structures in real life congestion situations such as manufacturing and production, computer and communication systems, service and distribution systems, etc. In a queueing system with server vacations: vacation may start when the queue is empty or may start when there are customers in the queue. In literature, a time interval when the server is either unavailable (for various reasons) or idle is called a Vacation period. In Exhaustive service and multiple vacation policy the server keeps serving customers until the system is empty and then takes vacations for as long as the system is empty. Non-Exhaustive service policy refers to those systems, where the server may go on vacation even if there are some customers waiting for service. It is assumed that the server completes the service in hand before the interruption. Recently Indra \& Vijay [7] obtained the explicit transient solution of two-state markovian queuing model with exhaustive and nonexhaustive service in which arrivals or departures or both are occurring in batches of variable sizes.
However, in Exhaustive service and multiple vacation policy, the server stops the service completely during the vacation period. Past, Servi \& Finn [9] first studied an $\mathrm{M} / \mathrm{M} / 1$ queue with the working vacation policy: the server can work at different rate during the vacation period rather than stopping completely. Subsequently, Kim, Choi \& Chae [6], Wu \& Takagi [10] generalized results in [9] to an $\mathrm{M} / \mathrm{G} / 1$ queue with working vacations. Baba [1] extended this study to an GI/M/1 queue with working vacations by the matrix-analysis method. Banik et. al [2] analyzed the $\mathrm{GI} / \mathrm{M} / 1 / \mathrm{N}$ queue with working vacations. All of the above mentioned contributions on working vacation are confined to results describing steady-state operation only.

\section{MODEL DESCRIPTION}

In the present work, we study the transient solution of "Two-dimensional state markovian queueing system with multiple working vacations \& Non-Exhaustive service policy". We are following Pegden \& Rosenshine [8] (who analyzed the $M / M / 1$ queueing system in which the state of the system is given by $(i, j)$, where ' $i$ ' is the number of arrivals and ' $j$ ' is the number of departures until time $t$ ) along with the concept of working vacation, and also considering the Non-Exhaustive service policy, we obtained

1) Explicit probabilities of exact number of arrivals \& departures by a given time

2) Number of units arrive by time $t$

3) Numbers of units depart by time $t$ and many other related information.

The numerical results are provided for the above three situations. Finally particular cases of interest are derived there from.

The queueing system investigated in the present paper assumes that during the working vacation period and otherwise also the units are arriving in Poisson stream with parameter $\lambda$. The service times are exponentially distributed with parameters $\mu_{\mathrm{B}} \& \mu_{\mathrm{V}}$ for busy period and vacation period respectively. The availability time and vacation time of service channel follow exponential 
distribution with parameters $\mathrm{v}$ and $\mathrm{w}$ respectively. The various stochastic processes involved in the system are statistically independent \& initially the system starts with zero units and the server is on working vacation.

\section{DEFINITIONS AND NOTATIONS}

$\mathrm{P}_{\mathrm{i}, \mathrm{j}, \mathrm{B}}(\mathrm{t})=$ The probability that there are exactly $\mathrm{i}$ arrivals and $\mathrm{j}$ departures by time $\mathrm{t}$ and the server is busy in relation to the queue; $j<i$.

$P_{i, j, V}(t)=$ The probability that there are exactly $i$ arrivals and $\mathrm{j}$ departures by time $\mathrm{t}$ and the server is on working vacation; $\mathrm{j} \leq \mathrm{i}$.

$P_{i, j, F}(t)=$ The probability that there are exactly $i$ arrivals and $\mathrm{j}$ departures by time $\mathrm{t}$ and the server is free in relation to the queue; $j<i$.

$P_{i, j}(t)=\quad$ The probability that there are exactly $I$ arrivals and $\mathrm{j}$ departures by time $\mathrm{t} ; \mathrm{j} \leq \mathrm{i}$.

$\delta_{i, j}= \begin{cases}1 ; & \text { when } \mathrm{i}=\mathrm{j} \\ 0 ; & \text { when } \mathrm{i} \neq \mathrm{j}\end{cases}$

Laplace transform of $\mathrm{F}(\mathrm{t})$

$$
\begin{aligned}
& \overline{\mathrm{F}}(\mathrm{s})=\int_{0}^{\infty} \mathrm{e}^{-\mathrm{st}} \mathrm{F}(\mathrm{t}) \mathrm{dt} \quad \operatorname{Re}(\mathrm{s})>0 \\
& \sum_{\alpha}^{\beta}=1 \text { when } \beta<\alpha \\
& \left.\overline{\mathrm{F}}_{\mathrm{m}_{1}, \mathrm{~m}_{2}, \mathrm{~m}_{3}}^{\mathrm{a}, \mathrm{s}, \mathrm{s}}\right)=\left(\frac{1}{(\mathrm{~s}+\mathrm{a})^{\mathrm{m}_{1}}(\mathrm{~s}+\mathrm{b})^{\mathrm{m}_{2}}(\mathrm{~s}+\mathrm{c})^{\mathrm{m}_{3}}}\right)
\end{aligned}
$$

The Laplace inverse of

$$
\begin{array}{r}
\frac{\mathrm{Q}(\mathrm{p})}{\mathrm{P}(\mathrm{p})} \text { is } \sum_{\mathrm{k}=1}^{\mathrm{n}} \sum_{\ell=1}^{\mathrm{m}_{\mathrm{k}}} \frac{\mathrm{t}^{\mathrm{m}_{\mathrm{k}}-\ell} \mathrm{e}^{\alpha_{\mathrm{k}} \mathrm{t}}}{\left(\mathrm{m}_{\mathrm{k}}-\ell\right) !(\ell-1) !} \times\left.\frac{\mathrm{d}^{\ell-1}}{\mathrm{dp}^{\ell-1}} \frac{\mathrm{Q}(\mathrm{p})}{\mathrm{P}(\mathrm{p})}\left(\mathrm{p}-\alpha_{\mathrm{k}}\right)^{\mathrm{m}_{\mathrm{k}}}\right|_{\mathrm{p}=\alpha_{\mathrm{k}}} \\
\alpha_{\mathrm{i}} \neq \alpha_{\mathrm{k}} \text { for } \mathrm{i} \neq \mathrm{k}
\end{array}
$$

where, $P(p)=\left(p-\alpha_{1}\right)^{m_{1}}\left(p-\alpha_{2}\right)^{m_{2}} \ldots\left(p-\alpha_{n}\right)^{m_{n}}$ and

$\mathrm{Q}(\mathrm{p})$ is polynomial of degree $<$

$$
\mathrm{m}_{1}+\mathrm{m}_{2}+\mathrm{m}_{3}+\ldots+\mathrm{m}_{\mathrm{n}}-1
$$
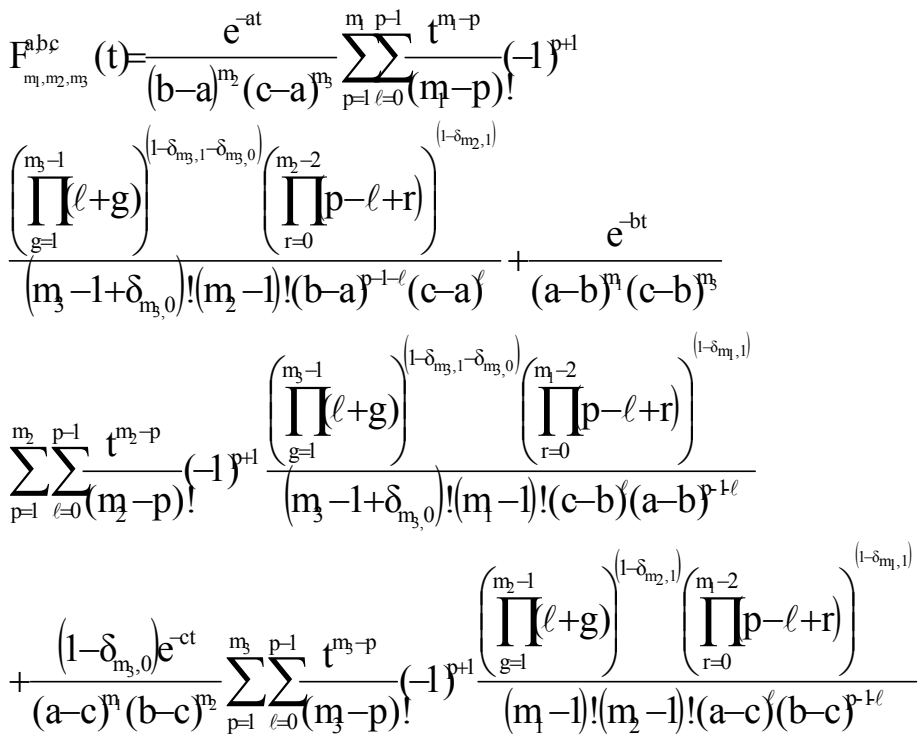

\section{SOLUTION OF THE PROBLEM} Initially

$$
\begin{aligned}
& \mathrm{P}_{0,0, \mathrm{~V}}(0)=1 \\
& \mathrm{P}_{0,0, \mathrm{~B}}(0)=0
\end{aligned}
$$

Difference-differential equations governing the system are

$$
\begin{gathered}
\frac{d}{d t} P_{i, j, V}(t)=-\left(\lambda+\mu_{V}+w\right) P_{i, j, V}(t)+\lambda P_{i-1, j, V}(t)+\mu_{V} P_{i, j-1, V}(t)\left(1-\delta_{j, 0}\right) \\
; i>j \geq 0 \\
\frac{d}{d t} P_{i, i, V}(t)=-\lambda P_{i, i, V}(t)+\mu_{V} P_{i, i-1, V}(t)\left(1-\delta_{i, 0}\right)+\mu_{B} P_{i, i-1, B}(t)\left(1-\delta_{i, 0}\right) \\
; i \geq 0
\end{gathered}
$$

$\frac{d}{d t} P_{i, j, B}(t)=-\left(\lambda+\mu_{B}\right) P_{i, j, B}(t)+\lambda P_{i-1, j, B}(t)\left(1-\delta_{i-1, j}\right)+w P_{i, j, V}(t)+v P_{i, j, F}(t)$

$$
; \mathrm{i}>\mathrm{j} \geq 0
$$

$$
\frac{d}{d t} P_{i, j, F}(t)=-(\lambda+v) P_{i, j, F}(t)+\lambda P_{i-1, j, F}(t)\left(1-\delta_{i-1, j}\right)+\mu_{B} P_{i, j-1, B}(t)
$$$$
; \mathrm{i}>\mathrm{j}>0
$$

Clearly,

$$
P_{i, j}(t)=P_{i, j, V}(t)+P_{i, j, B}(t)\left(1-\delta_{i, j}\right)+P_{i, j, F}(t)\left(1-\delta_{i, j}\right)
$$

Using (1) in eqns. (7) to (10) along with (5) \& (6) and solving recursively, we have

$$
\begin{aligned}
& \overline{\mathrm{P}}_{0,0, \mathrm{~V}}(\mathrm{~s})=\frac{1}{\mathrm{~s}+\lambda} \\
& \overline{\mathrm{P}}_{\mathrm{i}, 0, \mathrm{~V}}(\mathrm{~s})=\lambda^{\mathrm{i}} \overline{\mathrm{F}}_{1, \mathrm{i}}^{\lambda, \lambda+\mu_{\mathrm{V}}+\mathrm{w}}(\mathrm{s}) ; \mathrm{i}>0
\end{aligned}
$$




$$
\begin{aligned}
& \overline{\mathrm{P}}_{\mathrm{i}, \mathrm{j}, \mathrm{V}}(\mathrm{s})=\sum_{\mathrm{k}=\mathrm{j}}^{\mathrm{i}} \lambda^{\mathrm{i}-\mathrm{k}} \mu_{\mathrm{V}} \overline{\mathrm{F}}_{\delta_{\mathrm{k}, \mathrm{j}}, \mathrm{i}-\mathrm{k}+1-\delta_{\mathrm{k}, \mathrm{j}}}^{\lambda, \mu_{\mathrm{V}}+w} \overline{\mathrm{P}}_{\mathrm{k}, \mathrm{j}-1, \mathrm{~V}}(\mathrm{~s})+ \\
& \lambda^{\mathrm{i}-\mathrm{j}} \mu_{\mathrm{B}} \overline{\mathrm{F}}_{1, \mathrm{i}-\mathrm{j}}^{\lambda, \lambda+\mu_{\mathrm{v}}+w}(\mathrm{~s}) \overline{\mathrm{P}}_{\mathrm{j}, \mathrm{j}-1, \mathrm{~B}}(\mathrm{~s}) \\
& ; \mathrm{i} \geq \mathrm{j}>0 \\
& \overline{\mathrm{P}}_{\mathrm{i}, \mathrm{j}, \mathrm{F}}(\mathrm{s})=\sum_{\mathrm{k}=\mathrm{j}+1}^{\mathrm{i}}\left(\frac{\lambda}{\mathrm{s}+\lambda+\mathrm{v}}\right)^{\mathrm{i}-\mathrm{k}}\left(\frac{\mu_{\mathrm{B}}}{\mathrm{s}+\lambda+\mathrm{v}}\right) \overline{\mathrm{P}}_{\mathrm{k}, \mathrm{j}-1, \mathrm{~B}}(\mathrm{~s}) \\
& ; \mathrm{i}>\mathrm{j}>0 \\
& \overline{\mathrm{P}}_{\mathrm{i}, 0, \mathrm{~B}}(\mathrm{~s})=\sum_{\mathrm{k}=1}^{\mathrm{i}} \lambda^{\mathrm{i}} \mathrm{w} \overline{\mathrm{F}}_{1, \mathrm{i}-\mathrm{k}+1, \mathrm{k}}^{\lambda, \lambda+\mu_{\mathrm{B}}, \lambda+\mu_{\mathrm{v}}+\mathrm{w}}(\mathrm{s}) \\
& \text {;i }>0 \\
& \overline{\mathrm{P}}_{\mathrm{i}, \mathrm{j}, \mathrm{B}}(\mathrm{s})=\sum_{\mathrm{k}=\mathrm{j}+1}^{\mathrm{i}}\left(\frac{\lambda}{\mathrm{s}+\lambda+\mu_{\mathrm{B}}}\right)^{\mathrm{i}-\mathrm{k}}\left(\left(\frac{\mathrm{w}}{\mathrm{s}+\lambda+\mu_{\mathrm{B}}}\right) \overline{\mathrm{P}}_{\mathrm{k}, \mathrm{j}, \mathrm{V}}(\mathrm{s})\right. \\
& \left.+\left(\frac{\mathrm{v}}{\mathrm{s}+\lambda+\mu_{\mathrm{B}}}\right) \overline{\mathrm{P}}_{\mathrm{k}, \mathrm{j}, \mathrm{F}}(\mathrm{s})\right)
\end{aligned}
$$$$
; \mathrm{i}>\mathrm{j}>0
$$

The Laplace transform $\overline{\mathrm{P}}_{\mathrm{i}, \bullet}(\mathrm{s})$ of the probability $P_{i, \bullet}(t)$ that exactly $i$ units arrive by time $t$ is

$$
\overline{\mathrm{P}}_{\mathrm{i}, .}(\mathrm{s})=\sum_{\mathrm{j}=0}^{\mathrm{i}} \overline{\mathrm{P}}_{\mathrm{i}, \mathrm{j}}(\mathrm{s})=\frac{\lambda^{\mathrm{i}}}{(\mathrm{s}+\lambda)^{\mathrm{i}+1}} ; \mathrm{i} \geq 0
$$

The Laplace transform of the mean number of the arrivals is

$$
\sum_{\mathrm{i}=0}^{\infty} \mathrm{i} \overline{\mathrm{P}}_{\mathrm{i}, \bullet}(\mathrm{s})=\left(\frac{\lambda}{\mathrm{s}^{2}}\right)
$$

Using eqn. (4) for finding Laplace inverse of eqn. (12) to (17), we have

$$
\begin{aligned}
& \mathrm{P}_{0,0, \mathrm{~V}}(\mathrm{t})=\mathrm{e}^{-\lambda \mathrm{t}} \\
& \mathrm{P}_{\mathrm{i}, 0, \mathrm{~V}}(\mathrm{t})=\lambda^{\mathrm{i}} \mathrm{F}_{1, \mathrm{i}}^{\lambda, \lambda+\mu_{\mathrm{v}}+\mathrm{w}}(\mathrm{t}) ; \mathrm{i} \geq 0 \\
& \mathrm{P}_{\mathrm{i}, 0, \mathrm{~B}}(\mathrm{t})=\sum_{\mathrm{k}=1}^{\mathrm{i}} \lambda^{\mathrm{i}} \mathrm{w} \mathrm{F}_{1, \mathrm{i}-\mathrm{k}+1, \mathrm{k}}^{\lambda, \lambda+\mu_{\mathrm{v}}+\mathrm{w}}(\mathrm{t}) ; \mathrm{i}>0
\end{aligned}
$$

$$
\begin{aligned}
P_{i, j, V}(t)= & \sum_{k=j}^{i} \lambda^{i-k} \mu_{V} F_{\delta_{k, j}, i-k+1-\delta_{k, j}}^{\lambda, \lambda+\mu_{V}+w}(t) * P_{k, j-1, V}(t)+ \\
& \lambda^{i-j} \mu_{B} F_{1, i-j}^{\lambda, \lambda+\mu_{V}+w}(t) * P_{j, j-1, B}(t) \\
& ; i \geq j>0 \\
P_{i, j, F}(t)= & \sum_{k=j+1}^{i} \lambda^{i-k} \mu_{B} e^{-(\lambda+v) t} \frac{t^{i-k}}{(i-k) !} * P_{k, j-1, B}(t) \\
P_{i, j, B}(t)= & \sum_{k=j+1}^{i} \lambda^{i-k} w e^{-\left(\mu_{B}+\lambda\right) t} \frac{t^{i-k}}{(i-k) !} * P_{k, j, V}(t)+\sum_{k=j+1}^{i} \lambda^{i-k} v e^{-\left(\mu_{B}+\lambda\right) t} \\
& \frac{t^{i-k}}{(i-k) !} * P_{k, j, F}(t) \quad ; i>j>0(25)
\end{aligned}
$$

And

$$
\mathrm{P}_{\mathrm{i}, \bullet}(\mathrm{t})=\left(\frac{(\lambda \mathrm{t})^{\mathrm{i}}}{\mathrm{i} !} \mathrm{e}^{-\lambda \mathrm{t}}\right) ; \mathrm{i} \geq 0
$$

The arrivals follow Poisson distribution as the probability of total number of arrivals is not affected by the vacation time and availability time of the server.

The graphical representation of $P_{i, \bullet}(t)$ with the variation of arrival rate $(\lambda)$ has also been shown in the figures 1(a)-1(d).

The Laplace inverse of the mean number of arrivals by time $\mathbf{t}$

$$
\sum_{i=0}^{\infty} i P_{i, .}(t)=\lambda t
$$

From $(12)-(17)$, it is seen that

$$
\begin{array}{ll}
\sum_{i=0}^{\infty} \sum_{j=0}^{i} \bar{P}_{i, j}(s)=\frac{1}{s} & \text { and hence } \\
\sum_{i=0}^{\infty} \sum_{j=0}^{i} P_{i, j}(t)=1 & \text { a verification. }
\end{array}
$$

\section{PARTICULAR CASES}

Case I- When the server is following exhaustive service policy only i.e. letting $\mathrm{V} \rightarrow \infty$, we have

$$
\begin{aligned}
& \mathrm{P}_{0,0, \mathrm{~V}}(\mathrm{t})=\mathrm{e}^{-\lambda \mathrm{t}} \\
& \mathrm{P}_{\mathrm{i}, 0, \mathrm{~V}}(\mathrm{t})=\lambda^{\mathrm{i}} \mathrm{F}_{1, \mathrm{i}}^{\lambda, \lambda+\mu_{\mathrm{V}}+\mathrm{w}}(\mathrm{t}) ; \mathrm{i}>0
\end{aligned}
$$




$$
\begin{aligned}
& \mathrm{P}_{\mathrm{i}, \mathrm{j}, \mathrm{V}}(\mathrm{t})=\sum_{\mathrm{k}=\mathrm{j}}^{\mathrm{i}} \lambda^{\mathrm{i}-\mathrm{k}} \mu_{\mathrm{V}} \mathrm{F}_{\delta_{\mathrm{k}, j}, \mathrm{j}, \mathrm{k}+\mathrm{k}+1-\delta_{\mathrm{k}, \mathrm{j}}}^{\lambda \lambda+\mathrm{u}_{\mathrm{j}}+\mathrm{t}} * \mathrm{P}_{\mathrm{k}, \mathrm{j}-1, \mathrm{~V}}(\mathrm{t})+ \\
& \lambda^{\mathrm{i}-\mathrm{j}} \mu_{\mathrm{B}} \mathrm{F}_{1, \mathrm{i}-\mathrm{j}}^{\lambda \lambda \lambda+\mu_{\mathrm{v}}+\mathrm{w}}(\mathrm{t}) * \mathrm{P}_{\mathrm{j}, \mathrm{j}-1, \mathrm{~B}}(\mathrm{t}) \\
& ; \mathrm{i} \geq \mathrm{j}>0 \\
& \mathrm{P}_{\mathrm{i}, 0, \mathrm{~B}}(\mathrm{t})=\sum_{\mathrm{k}=1}^{\mathrm{i}} \lambda^{\mathrm{i}} \mathrm{w} \mathrm{F}_{1, \mathrm{i}-\mathrm{k}+1, \mathrm{k}}^{\lambda, \lambda+\mu_{\mathrm{B}}, \lambda+\mu_{\mathrm{v}}+\mathrm{w}}(\mathrm{t}) ; \mathrm{i}>0 \\
& P_{i, j, B}(t)=\sum_{k=j+1}^{i} \lambda^{i-k} w e^{-\left(\mu_{B}+\lambda\right) t} \frac{t^{i-k}}{(i-k) !} * P_{k, j, V}(t) \\
& ; \mathrm{i}>\mathrm{j}>0
\end{aligned}
$$

These results agree with eqns. (10) to (13) of Indra \& Ruchi [5].

Case II- Along with case -I, when server is on vacation only then the rate of doing work during vacation period is zero i.e. $\mu_{\mathrm{V}}=0$ in (30) to (34), we have

$$
\begin{aligned}
& \mathrm{P}_{0,0, \mathrm{~V}}(\mathrm{t})=\mathrm{e}^{-\lambda \mathrm{t}} \\
& \mathrm{P}_{\mathrm{i}, 0, \mathrm{~V}}(\mathrm{t})=\lambda^{\mathrm{i}} \mathrm{F}_{1, \mathrm{i}}^{\lambda, \lambda+\mathrm{w}}(\mathrm{t}) ; \mathrm{i} \geq 0 \\
& \mathrm{P}_{\mathrm{i}, \mathrm{j}, \mathrm{V}}(\mathrm{t})=\lambda^{\mathrm{i}-\mathrm{j}} \mu_{\mathrm{B}} \mathrm{F}_{1, \mathrm{i}-\mathrm{j}}^{\lambda, \lambda+w}(\mathrm{t}) * \mathrm{P}_{\mathrm{j}, \mathrm{j}-1, \mathrm{~B}}(\mathrm{t}) \\
& \mathrm{P}_{\mathrm{i}, 0, \mathrm{~B}}(\mathrm{t})=\sum_{\mathrm{k}=1}^{\mathrm{i}} \lambda^{\mathrm{i}} \mathrm{w}_{1, \mathrm{i}-\mathrm{k}+1, \mathrm{k}}^{\lambda, \lambda+\mathrm{w}, \lambda+\mu_{\mathrm{B}}+\mathrm{w}}(\mathrm{t}) ; \mathrm{i}>0 \\
& \mathrm{P}_{\mathrm{i}, \mathrm{j}, \mathrm{B}}(\mathrm{t})=\sum_{\mathrm{k}=\mathrm{j}+1}^{\mathrm{i}} \lambda^{\mathrm{i}-\mathrm{k}} \mathrm{we}^{-\left(\mu_{\mathrm{B}}+\lambda\right) \mathrm{t}} \frac{\mathrm{t}^{\mathrm{i}-\mathrm{k}}}{(\mathrm{i}-\mathrm{k}) !} * \mathrm{P}_{\mathrm{k}, \mathrm{j}, \mathrm{V}}(\mathrm{t})
\end{aligned}
$$

These results agree with eqns. (1.2.15) to (1.2.20) of Indra [4].

Case III- In continuation with case II, when server is instantaneously available and he does not go for a vacation i.e. the mean vacation time $\mathrm{w}^{-1}$ is zero. Letting $\mathrm{W} \rightarrow \infty$ in (35) to (39), we have

$$
\begin{aligned}
& \mathrm{P}_{0,0}(\mathrm{t})=\mathrm{P}_{0,0, \mathrm{~V}}(\mathrm{t})=\mathrm{e}^{-\lambda \mathrm{t}} \\
& \mathrm{P}_{\mathrm{i}, \mathrm{i}}(\mathrm{t})=\mathrm{P}_{\mathrm{i}, \mathrm{i}, \mathrm{V}}(\mathrm{t})=\left(\frac{\lambda}{\mu}\right)^{\mathrm{i}} \frac{(\mu \mathrm{t})^{\mathrm{i}} \mathrm{e}^{-\lambda \mathrm{t}}}{\mathrm{i} !} \sum_{\mathrm{K}=0}^{\mathrm{i}} \frac{(\mathrm{i}-\mathrm{k})}{\mathrm{k} !} \\
& \sum_{\mathrm{m}=0}^{\mathrm{i}-\mathrm{k}}\left(\frac{(-1)^{\mathrm{m}}(\mathrm{m}+\mathrm{i}+\mathrm{k}) !}{\mathrm{m} !(\mathrm{i}-\mathrm{k}-\mathrm{m}) !(\mu \mathrm{t})^{\mathrm{m}+\mathrm{k}}}\right)\left(1-\mathrm{e}^{-\mu \mathrm{t}} \sum_{\mathrm{r}=0}^{\mathrm{m}+\mathrm{i}+\mathrm{k}-1} \frac{(\mu \mathrm{t})^{\mathrm{r}}}{\mathrm{r} !}\right)
\end{aligned}
$$

$$
\text { ; }>0
$$

$$
\begin{aligned}
& P_{i, j}(t)=P_{i, j, B}(t)=\left(\frac{\lambda}{\mu}\right)^{i} \frac{(\mu t)^{j} e^{-\lambda t}}{i !} \sum_{K=0}^{j} \frac{(i-k)}{k !} \\
& \sum_{m=0}^{j-k}\left(\frac{(-1)^{m}(m+i+k) !}{m !(j-k-m) !(\mu t)^{m+k}}\right)\left(1-e^{-\mu t} \sum_{r=0}^{m+i+k-1} \frac{(\mu t)^{r}}{r !}\right)
\end{aligned}
$$

$$
; \mathrm{i} \geq \mathrm{j} \geq 0
$$

Eqns. (40) to (42) coincide with eqn. (5) of Pegden and Rosenshine [8].

\section{NUMERICAL RESULTS}

1. The numerical results for the probabilities of exact number of arrivals

(i) by a given time i.e. $\sum_{j=0}^{i} P_{i, j}(t)$

(ii) during busy period i.e. $\sum_{\mathrm{j}=0}^{\mathrm{i}} \mathrm{P}_{\mathrm{i}, \mathrm{j}, \mathrm{B}}(\mathrm{t})$

(iii) during vacation period i.e. $\sum_{j=0}^{i} P_{i, j, V}(t)$

(iv) during free period i.e. $\sum_{j=0}^{i} P_{i, j, F}(t)$

are computed for different sets of parameter and is summarized in Table - I. The Table - I shows complete agreement with the Table - I of Pegden \& Rosenshine [8] except the columns having probabilities of arrivals during busy period, vacation period and free period. All the computation works are performed on Pentium IV using MATLAB software.

Table-I is based on the relationship

$\operatorname{Pr}\{i$ arrivals in $(0, t)\}=\frac{e^{-\lambda t}(\lambda t)^{i}}{i !}=\sum_{j=0}^{\infty} P_{i, j}(t) \quad$ where $P_{i, j}(t)$ is defined in eqn.(11). 
Table - I

\begin{tabular}{|c|c|c|c|c|c|c|c|c|c|c|}
\hline$\lambda$ & $\mu_{\mathrm{B}}$ & $\mu_{v}$ & $\begin{array}{l}\text { W } \\
\& \\
V\end{array}$ & $\mathrm{t}$ & $\mathrm{i}$ & $\frac{e^{-\lambda t}(\lambda t)^{i}}{i !}$ & $\sum_{j=0}^{i} P_{i, j, B}(t)$ & $\sum_{j=0}^{i} P_{i, j, V}(t)$ & $\sum_{\mathrm{j}=0}^{\mathrm{i}} \mathrm{P}_{\mathrm{i}, \mathrm{j}, \mathrm{F}}(\mathrm{t})$ & $\sum_{\mathrm{j}=0}^{\mathrm{i}} \mathrm{P}_{\mathrm{i}, \mathrm{j}}(\mathrm{t})$ \\
\hline 1 & 2 & 1 & 1 & 3 & 1 & $\begin{array}{l}0.1493 \\
61\end{array}$ & $\begin{array}{l}0.0122 \\
30799\end{array}$ & $\begin{array}{l}0.1371 \\
30405\end{array}$ & 0.0 & $\begin{array}{l}0.1493612 \\
04\end{array}$ \\
\hline 1 & 2 & 1 & 1 & 3 & 3 & $\begin{array}{l}0.2240 \\
42\end{array}$ & $\begin{array}{l}0.0541 \\
04040\end{array}$ & $\begin{array}{l}0.1233 \\
45354\end{array}$ & $\begin{array}{l}0.0465 \\
92414\end{array}$ & $\begin{array}{l}0.2240418 \\
08\end{array}$ \\
\hline 1 & 2 & 1 & 1 & 3 & 5 & $\begin{array}{l}0.1008 \\
19\end{array}$ & $\begin{array}{l}0.0314 \\
79719\end{array}$ & $\begin{array}{l}0.0264 \\
36061\end{array}$ & $\begin{array}{l}0.0429 \\
03034\end{array}$ & $\begin{array}{l}0.1008188 \\
14\end{array}$ \\
\hline 2 & 2 & 1 & 1 & 3 & 1 & $\begin{array}{l}0.0148 \\
73\end{array}$ & $\begin{array}{l}0.0012 \\
17872\end{array}$ & $\begin{array}{l}0.0136 \\
54642\end{array}$ & 0.0 & $\begin{array}{l}0.0148725 \\
13\end{array}$ \\
\hline 2 & 2 & 1 & 1 & 3 & 3 & $\begin{array}{l}0.0892 \\
35\end{array}$ & & $\begin{array}{l}0.0491 \\
28028\end{array}$ & $\begin{array}{l}0.0185 \\
57598\end{array}$ & $\begin{array}{l}0.0892350 \\
78\end{array}$ \\
\hline 2 & 2 & 1 & 1 & 3 & 5 & $\begin{array}{l}0.1606 \\
23\end{array}$ & $\begin{array}{l}0.0501 \\
53054\end{array}$ & $\begin{array}{l}0.0421 \\
17566\end{array}$ & $\begin{array}{l}0.0683 \\
52521\end{array}$ & $\begin{array}{l}0.1606231 \\
41\end{array}$ \\
\hline 1 & 2 & 1 & 1 & 4 & 1 & $\begin{array}{l}0.0732 \\
63\end{array}$ & $\begin{array}{l}0.0045 \\
65085\end{array}$ & $\begin{array}{l}0.0686 \\
97470\end{array}$ & 0.0 & $\begin{array}{l}0.0732625 \\
55\end{array}$ \\
\hline 1 & 2 & 1 & 1 & 4 & 3 & $\begin{array}{l}0.1953 \\
67\end{array}$ & $\begin{array}{l}0.0400 \\
20126\end{array}$ & $\begin{array}{l}0.1233 \\
65030\end{array}$ & $\begin{array}{l}0.0319 \\
81659\end{array}$ & $\begin{array}{l}0.1953668 \\
15\end{array}$ \\
\hline 1 & 2 & 1 & 1 & 4 & 5 & $\begin{array}{l}0.1562 \\
93\end{array}$ & & & & $\begin{array}{l}0.1562934 \\
53\end{array}$ \\
\hline 2 & 2 & 1 & 1 & 4 & 3 & $\begin{array}{l}0.0286 \\
26\end{array}$ & $\begin{array}{l}0.0058 \\
63953\end{array}$ & $\begin{array}{l}0.0180 \\
76075\end{array}$ & $\begin{array}{l}0.0046 \\
86116\end{array}$ & $\begin{array}{l}0.0286261 \\
44\end{array}$ \\
\hline 2 & 2 & 1 & 1 & 4 & 5 & $\begin{array}{l}0.0916 \\
04\end{array}$ & $\begin{array}{l}0.0271 \\
97807\end{array}$ & $\begin{array}{l}0.0270 \\
78459\end{array}$ & $\begin{array}{l}0.0373 \\
27396\end{array}$ & $\begin{array}{l}0.0916036 \\
62\end{array}$ \\
\hline 2 & 4 & 2 & 1 & 4 & 5 & $\begin{array}{l}0.0916 \\
04\end{array}$ & $\begin{array}{l}0.0121 \\
98832\end{array}$ & $\begin{array}{l}0.0538 \\
88396\end{array}$ & $\begin{array}{l}0.0255 \\
16434\end{array}$ & $\begin{array}{l}0.0916036 \\
62\end{array}$ \\
\hline 1 & 2 & 1 & 1 & 4 & 4 & $\begin{array}{l}0.1953 \\
67\end{array}$ & $\begin{array}{l}0.0509 \\
81684\end{array}$ & $\begin{array}{l}0.0870 \\
27214\end{array}$ & $\begin{array}{l}0.0573 \\
57916\end{array}$ & $\begin{array}{l}0.1953668 \\
14\end{array}$ \\
\hline 1 & 2 & 1 & 1 & 3 & 6 & $\begin{array}{l}0.0504 \\
09\end{array}$ & $\begin{array}{l}0.0163 \\
21797\end{array}$ & $\begin{array}{l}0.0095 \\
90708\end{array}$ & $\begin{array}{l}0.0244 \\
96902\end{array}$ & $\begin{array}{l}0.0504094 \\
07\end{array}$ \\
\hline
\end{tabular}

2. The departure process from the $M / M / 1$ queue has the distribution function $P(j, t)$, the probability that exactly $j$ customers have been served by time $t$. In terms of $P_{i, j}(t)$, we have

$$
P(j, t)=\sum_{i=j}^{\infty} P_{i, j}(t) \quad \&
$$

$$
P(j, t)=P_{B}(j, t)+P_{V}(j, t)+P_{F}(j, t)
$$

where $P_{B}(j, t)=\sum_{i=j}^{\infty} P_{i, j, B}(t), \quad P_{V}(j, t)=\sum_{i=j}^{\infty} P_{i, j, V}(t)$ and $P_{F}(j, t)=\sum_{i=j}^{\infty} P_{i, j, F}(t)$

Tables II shows values of $P(j, t)$ respectively for different values of $t$. Table II coincides approximately with table I of
Hubbard et al. [3] because they computed 28 values \& we are able to compute only 7 values.

Figs. 2(a) - 2(d) display the effect of different values of $\lambda$ on $P_{B}(j, t), P_{V}(j, t), P_{F}(j, t) \& P(j, t)$.

Table - II

Values of $\mathrm{P}(\mathrm{j}, \mathrm{t})$ for $\lambda=1, \mu_{\mathrm{B}}=4, \mu_{\mathrm{V}}=4, \mathrm{v}=1 \& \mathrm{w}=1$

\begin{tabular}{|l|l|l|l|c|c|}
\hline \multicolumn{1}{|c|}{$\mathrm{j}$} & \multicolumn{1}{c|}{$\mathrm{t}=1$} & \multicolumn{1}{c|}{$\mathrm{t}=3$} & \multicolumn{1}{c|}{$\mathrm{t}=5$} & $\mathrm{t}=7$ & $\mathrm{t}=10$ \\
\hline 0 & 0.484397903 & 0.066376912 & 0.008983381 & 0.001215769 & 0.000060529 \\
\hline 1 & 0.371603904 & 0.198424464 & 0.046464401 & 0.008829504 & 0.000617739 \\
\hline 2 & 0.118475832 & 0.27111896 & 0.113871393 & 0.030870402 & 0.003128400 \\
\hline 3 & 0.02230139 & 0.226474024 & 0.174763531 & 0.068715478 & 0.010134541 \\
\hline 4 & 0.002857452 & 0.131597834 & 0.188280632 & 0.108802889 & 0.023693062 \\
\hline 5 & 0.000263886 & 0.05620838 & 0.149284734 & 0.128007415 & 0.041690377 \\
\hline 6 & 0.000016391 & 0.016290888 & 0.08053539 & 0.103269597 & 0.050810907 \\
\hline Total & $\mathbf{0 . 9 9 9 9 1 6 7 5 8}$ & $\mathbf{0 . 9 6 6 4 9 1 4 6 2}$ & $\mathbf{0 . 7 6 2 1 8 3 4 6 2}$ & $\mathbf{0 . 4 4 9 7 1 1 0 5 4}$ & $\mathbf{0 . 1 3 0 1 3 5 5 5 5}$ \\
\hline
\end{tabular}

3. The probability of exactly $n$ customers in the system at time $t$, denoted by $P(n, t)$ can be expressed in terms of $\mathrm{P}_{\mathrm{i}, \mathrm{j}}(\mathrm{t})$ as

$$
\begin{aligned}
& P(n, t)=\sum_{j=0}^{\infty} P_{j+n, j}(t) \quad \& \\
& P(n, t)=P_{B}(n, t)+P_{V}(n, t)+P_{F}(n, t)
\end{aligned}
$$

Where

$$
\begin{aligned}
& P_{B}(n, t)=\sum_{j=0}^{\infty} P_{j+n, j, B}(t), \quad P_{V}(n, t)=\sum_{j=0}^{\infty} P_{j+n, j, V}(t) \\
& \& \quad P_{F}(n, t)=\sum_{j=0}^{\infty} P_{j+n, j, F}(t)
\end{aligned}
$$

Values of $\mathrm{P}(\mathrm{n}, \mathrm{t})$ with parameters $\lambda=1, \mu_{\mathrm{B}}=2$, $\mu_{\mathrm{V}}=1, \mathrm{~V}=1$ and $\mathrm{W}=1$ for different values of $\mathrm{t}$ are shown in the following table.

Figs. 3(a) - 3(d) depict the effect of different values of $\lambda$ on $\mathrm{P}_{B}(\mathrm{n}, \mathrm{t}), \mathrm{P}_{V}(\mathrm{n}, \mathrm{t}), \mathrm{P}_{\mathrm{F}}(\mathrm{n}, \mathrm{t}) \& \mathrm{P}(\mathrm{n}, \mathrm{t})$.

Table III for $\mathrm{P}(\mathrm{n}, \mathrm{t})$

\begin{tabular}{|l|l|l|l|}
\hline $\mathbf{N}$ & $\mathbf{t}=\mathbf{1}$ & $\mathbf{t}=\mathbf{3}$ & $\mathbf{t}=\mathbf{5}$ \\
\hline 0 & 0.544463487 & 0.342393336 & 0.247722816 \\
\hline 1 & 0.301386032 & 0.244239753 & 0.21074915 \\
\hline 2 & 0.113474309 & 0.178057177 & 0.158116102 \\
\hline 3 & 0.031975805 & 0.126442243 & 0.0959276 \\
\hline 4 & 0.007149923 & 0.073748734 & 0.04049963 \\
\hline 5 & 0.001308813 & 0.025124171 & 0.008882113 \\
\hline 6 & 0.000103028 & 0.001350017 & 0.000372955 \\
\hline Total & $\mathbf{0 . 9 9 9 8 6 1 3 9 7}$ & $\mathbf{0 . 9 9 1 3 5 5 4 3 1}$ & $\mathbf{0 . 7 6 2 2 7 0 3 6 6}$ \\
\hline
\end{tabular}


4. The waiting time distribution for a customer can be derived as $\mathrm{P}(\mathrm{W}>\tau \mid \mathrm{t})$, the probability that a customer waits more than $\tau$ time units in the system, given that the customer arrives at time $\mathrm{t}$ is based on the relationship $=$

$\sum_{n=0}^{\infty} \mathrm{P}($ number of services by time $\tau<\mathrm{n}+1) \mathrm{P}_{\mathrm{n}}(\mathrm{t})$

$$
=\mathrm{e}^{-\mu \tau} \sum_{\mathrm{n}=0}^{\infty} \sum_{\mathrm{s}=0}^{\mathrm{n}} \frac{(\mu \tau)^{\mathrm{s}}}{\mathrm{s} !} \mathrm{P}_{\mathrm{n}}(\mathrm{t})
$$

Fig. 4 depicts the effect of waiting time on the system.

5. The cumulative distribution for the sojourn time in the system is

$\mathrm{P}(\mathrm{W} \leq \tau \mid \mathrm{t})=1-\mathrm{e}^{-\mu \tau} \sum_{\mathrm{n}=0}^{\infty} \sum_{\mathrm{s}=0}^{\mathrm{n}} \frac{(\mu \tau)^{\mathrm{s}}}{\mathrm{s} !} \mathrm{P}_{\mathrm{n}}(\mathrm{t})$

The graphical representation has also been shown in the fig. -5 .

6. The system utilization, i.e. the fraction of time the server is busy until time $t$ can also be expressed in terms of $P_{i, j}(t)$. Thus the fraction of the time the system is empty and consequently the server is on working vacation is $U_{v}(t)=\sum_{i=0}^{\infty} \sum_{j=0}^{i} P_{i, j, V}(t)$

and the fraction of time that the system is non-empty and hence the server utilized is

$$
\mathrm{U}_{\mathrm{B}}(\mathrm{t})=\sum_{\mathrm{i}=0}^{\infty} \sum_{\mathrm{j}=0}^{\mathrm{i}} \mathrm{P}_{\mathrm{i}, \mathrm{j}, \mathrm{B}}(\mathrm{t})^{\prime} \quad \mathrm{U}_{\mathrm{F}}(\mathrm{t})=\sum_{\mathrm{i}=0}^{\infty} \sum_{\mathrm{j}=0}^{\mathrm{i}} \mathrm{P}_{\mathrm{i}, \mathrm{F}, \mathrm{F}}(\mathrm{t})
$$

also total utilization time of server is given by $\mathrm{U}(\mathrm{t})=\mathrm{U}_{\mathrm{B}}(\mathrm{t})+\mathrm{U}_{\mathrm{V}}(\mathrm{t})+\mathrm{U}_{\mathrm{F}}(\mathrm{t})$

Table - IV is based on the above relationships and the graphical representation has also been shown in the fig. $-6(a), 6(b)$ and 6(c).

\section{Table -IV}

For $\lambda=1, \mu_{\mathrm{V}}=1, \mu_{\mathrm{B}}=2, \mathrm{w}=1$

\begin{tabular}{|c|l|l|l|l|}
\hline $\mathrm{t}$ & $\begin{array}{l}\text { Utilization } \\
\text { time during } \\
\text { busy period } \\
\mathrm{U}_{\mathrm{B}}(\mathrm{t})\end{array}$ & $\begin{array}{l}\text { Utilization } \\
\text { time during } \\
\text { working } \\
\text { vacation } \\
\text { period } \mathrm{U}_{\mathrm{V}}(\mathrm{t})\end{array}$ & $\begin{array}{l}\text { Utilization } \\
\text { time during } \\
\text { Non- } \\
\text { exhaustive } \\
\text { period } \mathrm{U}_{\mathrm{F}}(\mathrm{t})\end{array}$ & $\begin{array}{l}\text { Total } \\
\text { Utilization } \\
\text { time } \mathrm{U}(\mathrm{t})\end{array}$ \\
\hline 1 & $\begin{array}{l}0.119302785 \\
93942\end{array}$ & $\begin{array}{l}0.8511996743 \\
5319\end{array}$ & $\begin{array}{l}0.029414298 \\
55671\end{array}$ & $\begin{array}{l}0.999916758 \\
84932\end{array}$ \\
\hline 2 & 0.180126074 & 0.6984725393 & 0.116867580 & 0.995466194 \\
& 94299 & 3428 & 19665 & 47391 \\
\hline 3 & 0.200406256 & 0.5785167131 & 0.187568494 & 0.966491464 \\
767589 & 7672 & 69852 & 69113 \\
\hline 4 & 0.194568484 & 0.4826710870 & 0.212086450 & 0.889326021 \\
& 37424 & 6427 & 15892 & 59743 \\
\hline 5 & 0.168001955 & 0.4007713133 & 0.193410193 & 0.762183462 \\
& 63710 & 6457 & 97126 & 97294 \\
\hline
\end{tabular}
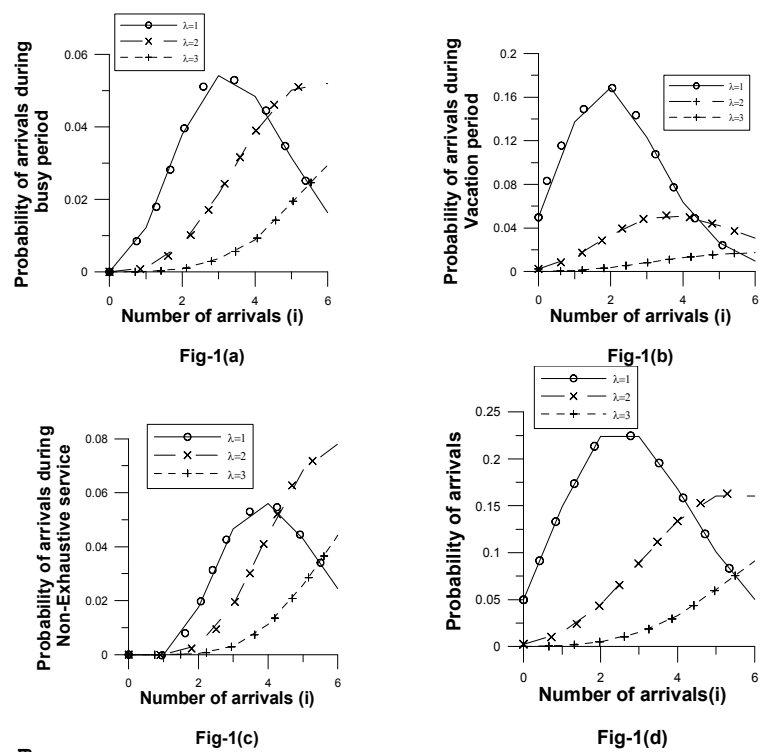

Fig-1(d)
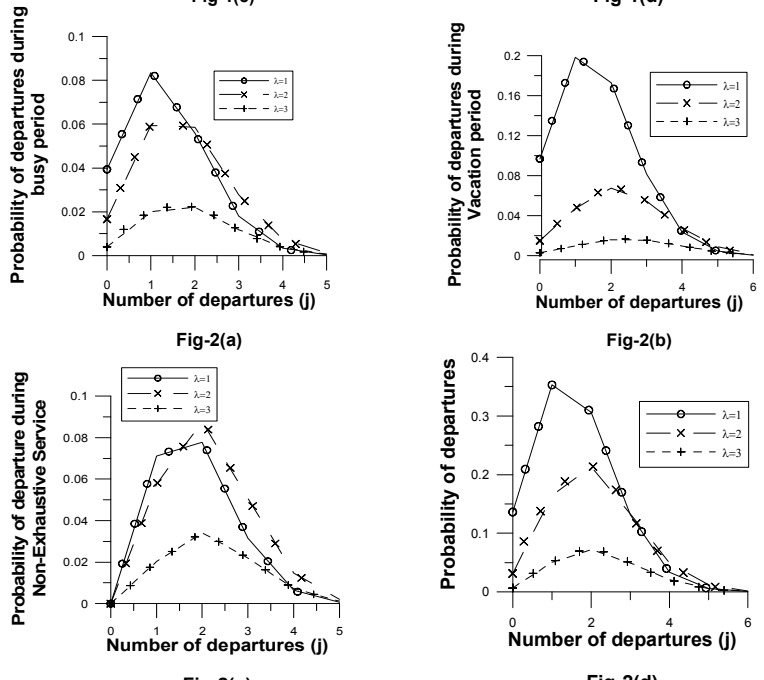

Fig-2(b)

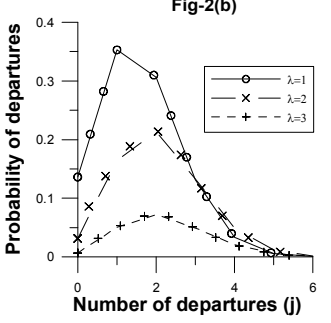

Fig-2(d)

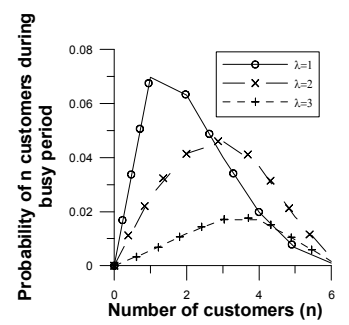

Fig-3(a)

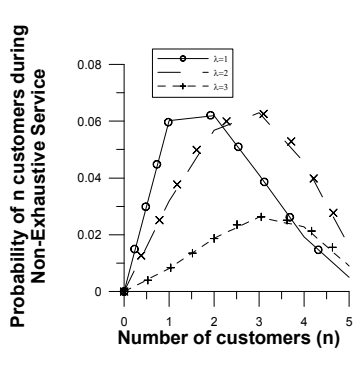

Fig-3(c)

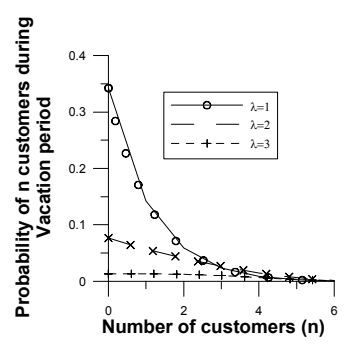

Fig-3(b)

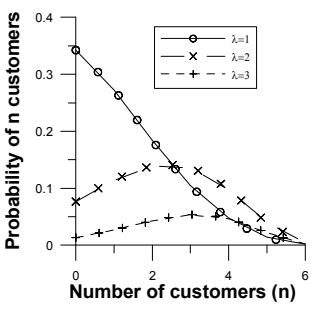

Fig-3(d) 

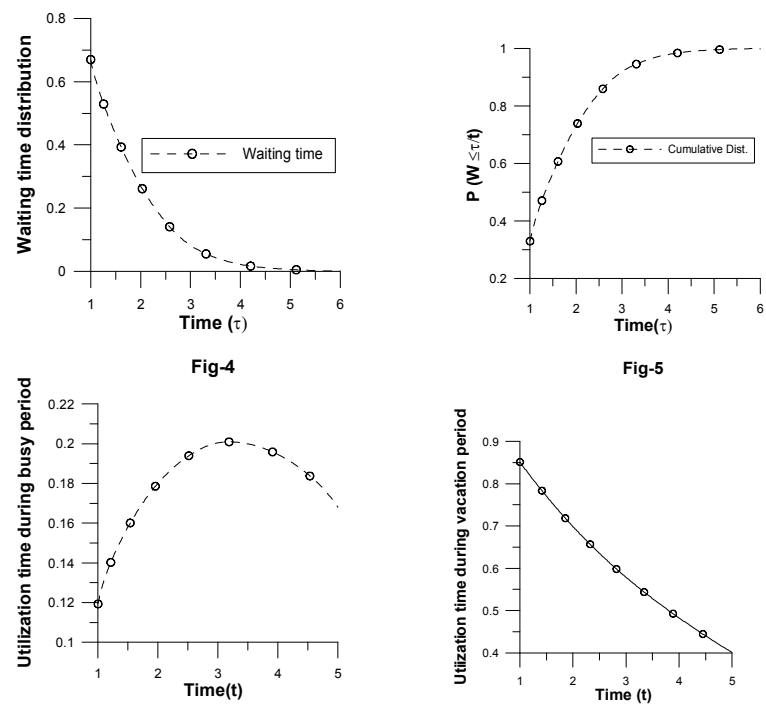

Fig-5
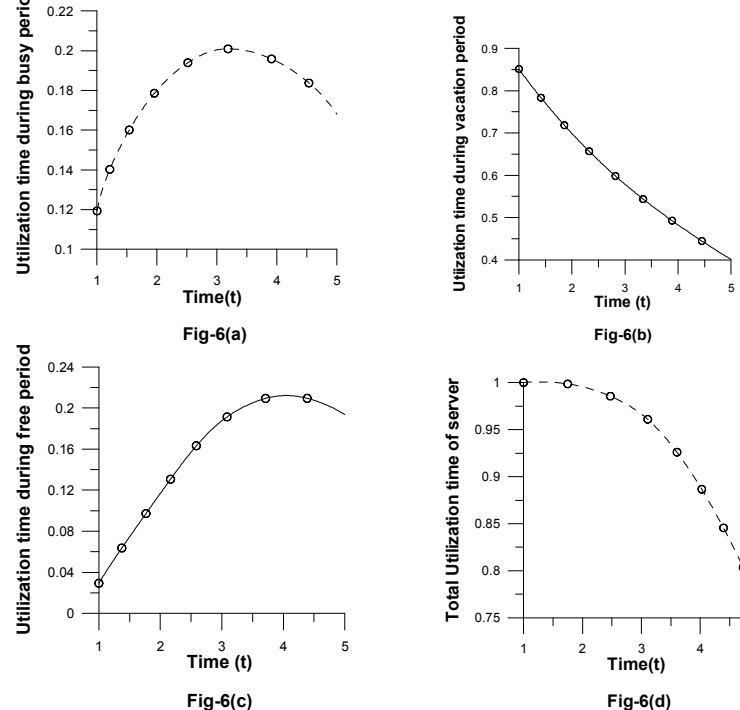

Fig-6(b)

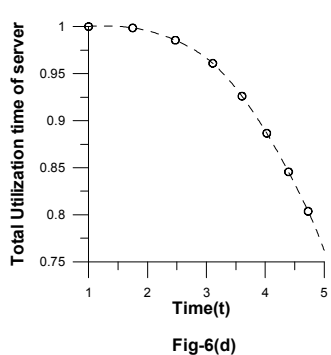

\section{ACKNOWLEDGEMENTS}

We are grateful to the Chairperson, Department of Statistics \& Operational Research, Kurukshetra University, Kurukshetra for providing necessary facilities and showing keen interest in this work.

\section{REFERENCES}

1. Baba, Y., (2005), Analysis of a GI/M/1 queue with multiple working vacations. Oper. Res. Lett. 33, 201209.

2. Banik, A.D., Gupta, U.C., Pathak, S.S. (2007), On the GI/M/1/N queue with multiple working vacations analytic analysis and computation Appl. Math. Modell. 31(9), 1701-1710.

3. Hubbard, J.R., Pegden, C.D. and Rosenshine, M. (1986), The departure process for the $M / M / 1$ queue, Journal of Applied Probability, Vol. 23, No. 1, (Mar.,1986), pp.249-255.

4. Indra, (1994), Some two-state single server queueing models with vacation or latest arrival run, Ph.D. thesis, Kurukshetra University, Kurukshetra.

5. Indra and Ruchi, (2009), Transient Analysis of TwoDimensional M/M/1 Queueing System with working vacations, Journal of Mathematics and System Science, Vol. 5, No. 2, (Dec. 2009) pp. 110-128.

6. Kim, J.D., Choi, D.W., Chae, K.C, (2003), Analysis of queue-length distribution of the $\mathrm{M} / \mathrm{G} / 1$ queue with working vacations In: Hawaii International Conference on Statistics and Related Fields.

7. Kumar Vijay, (2007), Two-State Bulk Queueing models with multiple vacations, Ph.D. Thesis, K.U. Kurukshetra.

8. Pegden, C.D. and Rosenshine, M. (1982), Some new results for the M/M/1 queue, Mgt Sci 28, 821-828 (1982).

9. Servi, L.D. and Finn, S.G., (2002), M/M/1 queues with working vacations (M/M/1/WV), Performance Evaluation, Vol. 50, pp 41-52.

10. Wu, D., Takagi, H., (2006), M/G/1 queue with multiple working vacations. Perform. Eval. 63(7), 654-681. 